\title{
Modelación Multivariada de la Satisfacción de Usuarios de Salud Primaria como Influencia del Pensamiento de Diseño (Design Thinking)
}

Segundo R. Cabana(1)*, Juan J. Montero(2) y Mauricio I. Aguilera(2)

(1) Facultad de Ingeniería, Dpto. de Ing. Industrial, Casilla 554, La Serena-Chile. (e-mail: rcabana@userena.cl)

(2) Universidad de La Serena, La Serena-Chile. (e-mail: jjmontero.investigacion.dii.uls@gmail.com, maguilera@userena.cl)

* Autor a quien debe ser dirigida la correspondencia

Recibido May. 10, 2019; Aceptado Jul. 8, 2019; Versión final Ago. 23, 2019, Publicado Dic. 2019

\begin{abstract}
Resumen
El objetivo del trabajo es desarrollar un modelo multivariado que permita analizar la influencia del pensamiento de diseño (design thinking) en la satisfacción de los usuarios de centros de salud familiar municipal (CESFAM), de la comuna de La Serena, Chile. Se construyó un modelo relacional aplicándose 361 encuestas a trabajadores. El análisis del modelo empleó métodos de ecuaciones estructurales, basado en los mínimos cuadrados parciales. Se determinó que la actitud de los directivos, la participación organizacional y los procesos formales de innovación, explican de forma directa y positiva en un $64,1 \%$ al pensamiento de diseño. La eficiencia y eficacia de los procesos, y la influencia del pensamiento de diseño en ellos, logran explicar en un $69,7 \%$ la satisfacción del usuario. En conclusión, los CESFAM deben enfocarse en instalar la metodología del pensamiento de diseño, a través de directivos que impulsen la participación organizacional, integrando además a sus usuarios y centrándose en la formalización de procesos innovadores.
\end{abstract}

Palabras clave: pensamiento de diseño; satisfacción del usuario; actitud directiva; centros de salud primaria; ecuaciones estructurales.

\section{Multivariate Modelling of the Satisfaction of Primary Health Users as an Influence of Design Thinking}

\begin{abstract}
The objective of the work is to develop a multivariate model that allows analyzing the influence of design thinking on the satisfaction of the users of municipal family health centers (CESFAM), in the city of La Serena, Chile. A relational model was constructed by applying a survey to 361 workers. The analysis of the model used methods of structural equations, based on partial least squares. It was determined that the attitude of the managers, the organizational participation and the formal processes of innovation, explain directly and positively the design thinking in a $64.1 \%$. The efficiency and effectiveness of the processes, and the influence of design thinking in them, manage to explain user satisfaction by $69.7 \%$. In conclusion, the CESFAM should focus on installing the methodology of design thinking, through managers that promote organizational participation, also integrating its users and focusing on the formalization of innovative processes.
\end{abstract}

Keywords: design thinking; user satisfaction; management attitude; primary health centers; structural equations 


\section{INTRODUCCIÓN}

En la actualidad, la mayoría de las empresas buscan generar valor mediante la sostenibilidad y la ventaja competitiva, a través de una gestión adecuada del talento de su capital humano, con una visión amplia y holística, logrando resolver problemas de costo, escasez y satisfacción integral de necesidades, permitiendo una mayor percepción de calidad de servicio por parte del cliente (Castillo et al., 2014). En este contexto, los métodos de mejora de calidad en salud han aumentado en popularidad como un medio clave por el cual las organizaciones priorizan sus recursos satisfaciendo las necesidades de los pacientes (Bhui, 2017).

A nivel internacional la calidad de la gestión de salud se ha centrado en programas formativos de educación médica basados en la ética, en este ámbito la Academia Americana de Médicos de Familia han elaborado unas directrices curriculares sobre competencias éticas que van dirigidas a los profesionales de medicina de familia. Estas competencias son fundamentales para el ejercicio de la medicina familiar y deben alcanzarse a través de una experiencia longitudinal que debe evaluar el consejo de acreditación para educación médica graduada. El objetivo de la educación médica incluye la adquisición de la reflexión crítica y los valores éticos que van a guiar al médico en su praxis asistencial, proporcionando una atención médica de alta calidad (PérezGarcía, 2017). Wintrup (2015), propone que la educación ética de los médicos en formación debe ir encaminada a la formación como agentes morales; es decir, al tratar de restituir la salud a un paciente deberán comportarse, primariamente, como un agente moral, y todas las decisiones que deberán tomar en el ejercicio de su profesión tendrán, indudablemente, un trasfondo ético.

La gestión de la salud en Chile ha desarrollado importantes innovaciones que son destacables a nivel mundial, como es la creación de las instituciones de salud previsional, permitiendo el acceso a los pacientes a una amplia gama de servicios de salud privados a través de libre elección y que finalmente derivó en el sistema mixto de atención que hoy (Clínicas de Chile A.G., 2009). Sin embargo, también se ha producido un aumento en los costos efectivos y utilitarios; por lo que la calidad es mirada desde la perspectiva de los prestadores de salud y no desde los pacientes, en quienes se deberían enfocar todos los esfuerzos. Un claro ejemplo de esto es el hecho de que las mejoras en procedimientos de cirugía, dispositivos de monitoreo en el hogar y la creación de nuevos productos médicos y medicina individualizada a personas en base a su genética no satisfacen las necesidades de los usuarios, haciéndose patente en el descontento con el servicio que ofrecen los diversos centros hospitalarios y consultorios, a tal nivel que incluso, la percepción del servicio en hospitales y consultorios son comparados con recintos carcelarios (Pezoa, 2011).

Kessler et al. (2017), sostienen que la innovación ha adquirido una importancia cada vez mayor en la atención de la salud, lo que hace urgente estructurar una configuración virtuosa de factores que permitan promover la innovación dentro de los organismos públicos, esto es, mediante el requerimiento de nuevas capacidades y competencias flexibles para la creación de nuevos conocimientos, que permitan generar un mejor aprovechamiento de recursos y una mejor calidad de los servicios de salud en base a las reales necesidades de los usuarios de los servicios hospitalarios, acorde a los resultados esperables de la incorporación de la creatividad e innovación dentro de la gestión de la salud en Chile, desafío por superar no sólo urgente, sino estratégico para asegurar la sostenibilidad de los centros de salud en Chile y el mundo, más aún si se considera que la percepción de gozar de buena salud ubica a Chile entre el $25 \%$ de los países peor clasificados de la Organización para la Cooperación y el Desarrollo Económico (OCDE, 2016). Una alternativa para abordar estas contingencias es el uso del pensamiento de diseño, el cual está enfocado a responder problemáticas complejas y que gracias a su metodología y paradigmas inherentes permite crear soluciones sinérgicas mediante la estructuración de equipos de trabajo multidisciplinarios que aborden problemáticas desde diversas perspectivas, creando soluciones innovadoras acorde a los requerimientos de los usuarios y en especial resolviendo problemas de costos y escases de recursos, que son factores que afectan la calidad y la forma en que la salud pública del país es brindada (Brown, 2008).

En este contexto, el estudio que se presenta se basa en nueve hipótesis relacionadas con los conceptos de actitud del equipo directivo, estrategias centradas en innovación, participación organizacional, actitud del personal, procesos formales de innovación, pensamiento de diseño, eficiencia, eficacia, y satisfacción presente en los centros de salud primaria de Chile. Hipótesis $1\left(\mathrm{H}_{1}\right)$ : Una actitud positiva de las jefaturas de área hacia la innovación facilita una organización orientada al pensamiento de diseño; Hipótesis $2\left(\mathrm{H}_{2}\right)$ : La implementación de una estrategia de innovación facilita una orientación al pensamiento de diseño; Hipótesis $3\left(\mathrm{H}_{3}\right)$ : La participación organizacional de diversos niveles en la propuesta de ideas innovadoras facilita una orientación al pensamiento de diseño; Hipótesis $4\left(\mathrm{H}_{4}\right)$ : Una actitud positiva del personal hacia la innovación facilita la orientación al pensamiento de diseño; Hipótesis $5\left(\mathrm{H}_{5}\right)$ : Un proceso formal de innovación facilita la orientación al pensamiento de diseño de una organización; Hipótesis $6\left(\mathrm{H}_{6}\right)$ : El pensamiento de diseño impacta positivamente en la eficacia de los procesos de atención en los centros de salud familiar; Hipótesis 7 ( $\left.\mathrm{H}_{7}\right)$ : El pensamiento de diseño impacta positivamente en la eficiencia de los procesos de atención en los centros de salud familiar; Hipótesis $8\left(\mathrm{H}_{8}\right)$ : La eficacia en los procesos de atención innovadores genera un impacto 
positivo en la satisfacción del usuario, e Hipótesis $9\left(\mathrm{H}_{9}\right)$ : La eficiencia en los procesos de atención innovadores genera un impacto positivo en la satisfacción del usuario

\section{ANTECEDENTES}

En primer lugar, se realiza una revisión de la literatura focalizado en las relaciones de variables; relación entre actitud del equipo directivo y el pensamiento de diseño, relación entre estrategias y el pensamiento de diseño, relación entre participación organizacional y pensamiento de diseño, relación entre actitud del personal y pensamiento de diseño, relación entre procesos formales de innovación y pensamiento de diseño, relación entre pensamiento de diseño y efectividad, relación entre eficacia y satisfacción de usuario, y relación entre eficiencia y satisfacción de usuario.

\section{Relación entre actitud del equipo directivo y el pensamiento de diseño}

La actitud del equipo directivo está representada por la apreciación de los directivos ante la promoción de nuevas ideas que pavimentan el camino para la orientación al pensamiento de diseño (Brown, 2008). En este aspecto, el pensamiento de diseño es, en su esencia, un proceso de innovación sistemática que prioriza la profunda empatía por los deseos, necesidades y desafíos del usuario final, para comprender plenamente un problema con la esperanza de desarrollar soluciones más integrales y efectivas (Roberts et al., 2016). La importancia de directivos radica en que son quienes definen y guían los esfuerzos organizacionales hacia la innovación y su gestión creando contextos organizacionales acordes y estableciendo estructuras, estrategias y sistemas alineados a la orientación al diseño y donde el apoyo y el reconocimiento brindado por el equipo directivo es lo que detona un proyecto de cambio (Nikulin et al., 2011). El apoyo de la alta dirección es considerado un factor crucial al momento de gestionar cualquier tipo de cambio dentro de las organizaciones, en esta línea la innovación y su gestión dentro de la empresa debe ser realizada con el apoyo de la alta administración, vale decir, los líderes de ellas para potenciar la gestión del conocimiento interno (Miranda y Astorga, 2013).

\section{Relación entre estrategias y el pensamiento de diseño}

La orientación al pensamiento de diseño se da con mayor recurrencia en una empresa con una estrategia clara de innovación, que impregna a la organización y fija objetivos claros que guían la administración de las inversiones y los esfuerzos para sus logros (Dülfer, 2014). La estrategia de innovación está orientada a ayudar a una empresa, a ser la primera en introducir productos o servicios heterogéneos en el mercado que tengan el potencial de moldear las preferencias y el comportamiento de los consumidores, una extensiva estrategia de innovación afecta la importancia otorgada no sólo a los proyectos de innovación que genera, sino también a los métodos que se involucran, incluyendo aquellos que afectan a la empresa hacia el diseño ofreciendo apoyo técnico e instrumental que, específicamente en ambientes innovadores, debe ser más flexible y tolerante a la incertidumbre, reforzando el rendimiento creativo y facilitando la innovación en general (Da Costa et al., 2014). Es por esto que la integración del pensamiento de diseño debe estar compuesto por acciones sistémicas e intencionadas, con directa relación al entorno de la organización y teniendo en cuenta el objetivo de mejorar la situación existente, a través de la innovación (Miranda y Astorga, 2013).

\section{Relación entre participación organizacional y pensamiento de diseño}

La orientación al diseño de pensamiento se asocia directamente con la participación organizacional y su innovación, lo que representa un involucramiento simultaneo de diferentes funciones y departamentos en procesos de innovación, es así que un clima organizacional que motiva e involucra a los empleados tiene un impacto positivo en el desempeño de sus actividades (Shanker et al., 2017). La orientación al pensamiento de diseño provoca que exista una alta cohesión nutrida de comunicación interna y externa, que posibilita el desarrollo de la creatividad, involucrando discusiones de empleados y testeo de nuevas opciones desde diversos puntos de vista, rompiendo con los moldes convencionales del creador en solitario y por tanto realizando gestiones adecuadas del conocimiento para potenciar la discusión en la organización, la cual refuerza la innovación y nutre de nuevo conocimiento tanto a los trabajadores como a la empresa, aumentando dicho recurso, que es visto como el más importante que la empresa gestiona, dado que influye en la eficiencia y eficacia de los procesos y la satisfacción de los clientes (Da Costa et al., 2014).

\section{Relación entre actitud del personal y pensamiento de diseño}

Si las organizaciones son capaces de desarrollar un clima organizacional percibido como positivo por los individuos, esto es más probable que resulte en mayores niveles de motivación y compromiso de los empleados (Shanker et al., 2017). Es la propia organización quien debe gestionar la capacidad de innovación de sus empleados, mediante la facilitación de ambientes, estructuras y apoyo acordes a los lineamientos del pensamiento de diseño que propicien la autonomía de los equipos de trabajo y la confianza para la minimización de la resistencia al cambio al incorporar nuevas metodologías de gestión en los procesos 
(Nikulin et al., 2011). La administración necesita asegurarse de que el clima organizacional se encargue de animar, nutrir y aumentar la creatividad individual de los empleados. De hecho, la adopción de pensamiento de diseño y la aplicación de sus procesos de diseño y herramientas requieren que los miembros de la organización experimenten personalmente cuando se generan y mejoren nuevas ideas (Brown, 2008).

\section{Relación entre procesos formales de innovación y pensamiento de diseño}

La innovación es un proceso sistemático y sistémico permanente que lleva a las organizaciones a su mejora continua y es el resultado de otro proceso que es la eficiente gestión del conocimiento (Miranda y Astorga, 2013), instalando bases sostenibles para instalar pensamiento de diseño, sus herramientas y lo que es más relevante sus paradigmas. Los procesos formales centrados en innovación brindan objetivos claros a los miembros de la organización, dando sentido a su trabajo, canalizando los esfuerzos y motivándolos a mejorar su rendimiento en innovación (Da Costa et al., 2014). Por lo que un proceso de innovación más formal también instala los principios de pensamiento de diseño y sus herramientas (Carlgren, 2013). La formalización de procesos de innovación puede ayudar a sobrellevar la resistencia en una organización y por lo tanto facilitan la implementación de procesos de diseño en la organización, brindando confianza y seguridad a quienes la conforman (Nikulin et al., 2011).

\section{Relación entre pensamiento de diseño y efectividad}

El pensamiento de diseño es un enfoque que utiliza la sensibilidad del diseñador y sus métodos de resolución de problemas para satisfacer las necesidades de las personas de un modo tecnológicamente factible y comercialmente viable, involucrando un conocimiento cabal de las necesidades del cliente. Nebot et al., (2011) mencionan que el criterio de evaluación en servicios de salud corresponde al juicio sobre el valor o utilidad de una intervención, siendo definida también por el diccionario de salud pública como los esfuerzos dirigidos a determinar de forma sistemática y objetiva el impacto de las actividades realizadas para alcanzar objetivos de salud, teniendo en cuenta los recursos asignados. La efectividad para efectos de evaluación y objetivos es un término de mayor alcance que la eficacia y la eficiencia, pues expresa la medida del impacto que un proceso determinado tiene sobre la salud de la población (Lam y Hernández, 2008).

\section{Relación entre eficacia y satisfacción de usuario}

La eficacia en servicios de salud es el grado en que un procedimiento o servicio puede lograr o mejorar el resultado posible, estando estos bajo condiciones ideales, las cuales difieren de acuerdo con las realidades locales y a los presupuestos que maneja cada centro de atención primaria de salud de la comuna (Lam y Hernández, 2008). Las personas eficaces son capaces de comprender mejor los roles requeridos en la realización de un comportamiento y de coordinar las habilidades necesarias (McKee et al., 2006). Las compañías orientadas al pensamiento de diseño están más propensas a mostrar mayores grados de eficacia al innovar en procesos de atención, debido a que al enfocarse en las experiencias y necesidades de los usuarios, el enfoque es una herramienta natural para innovar en el servicio, disminuyendo las brechas entre expectativas del usuario sobre un proceso de atención y su rendimiento al ser aplicado, lo que en definitiva provocaría responder de forma oportuna a los requerimientos de las personas. Dülfer (2014) señala que las compañías que ven las necesidades de los usuarios como una parte integral de sus operaciones tienen más probabilidad de lanzar nuevos productos y servicios y aproximadamente son doblemente más probables de mostrar un rápido crecimiento en sus resultados en términos de eficacia en el cumplimiento de expectativas ante el servicio.

\section{Relación entre eficiencia y satisfacción de usuario}

Según Oliver (1997) la satisfacción es la respuesta a la realización del consumidor. Es un juicio a las características de un producto/servicio, o al producto o servicio en sí, proporcionando un nivel de satisfacción relacionados con el consumo. La eficiencia se convierte en un desafío para estrategia de diversas organizaciones, quienes deben satisfacer las necesidades de sus usuarios y al mismo tiempo administrar óptimamente sus recursos. En ámbitos de salud pública, la eficiencia consiste justamente en un buen uso de recursos (Lam y Hernández, 2008), la que es evaluada mediante la valoración de los servicios y las actividades que se generan a partir estos, lo que implica que, si se obtienen mejores resultados con mejor gasto de recursos o menores esfuerzos, se habrá incrementado la eficiencia (Nebot et al., 2011). Lograr eficiencia en salud, significa también alcanzar los mejores resultados con los recursos disponibles, de modo que cuando se persiguen determinados resultados también deben quedar claras cuales son las formas más eficientes de alcanzarlos y qué procesos técnicos se deben abordar para llegar a ellos con eficiencia (Lam y Hernández, 2008).

Las herramientas del pensamiento de diseño extienden los límites de las soluciones propuestas ya que unifica el lenguaje entre equipos interdisciplinarios en torno a las necesidades del cliente, asegurándose de que cada miembro del equipo entienda que el resultado innovador final será posible (Dülfer, 2014), es así como los problemas potenciales de las formas tradicionales de innovar (que no consideran el prototipado iterativo) 
pueden ser superados ya que con esta metodología se detectarían tempranamente los problemas en la satisfacción del usuario, reduciéndose el riesgo de volver a desarrollar completamente una solución al no ser testeada en fases iniciales, lo que aumenta la probabilidad de cumplir con los tiempos, expectativas y costos (Carlgren, 2013).

\section{MODELO CAUSAL}

Considerando las relaciones anteriores, se construyó un modelo causal que busca identificar las variables que influyen en la satisfacción del usuario por medio de la técnica de pensamiento de diseño para atención primaria de salud en la región de Coquimbo, Chile (Fig. 1).

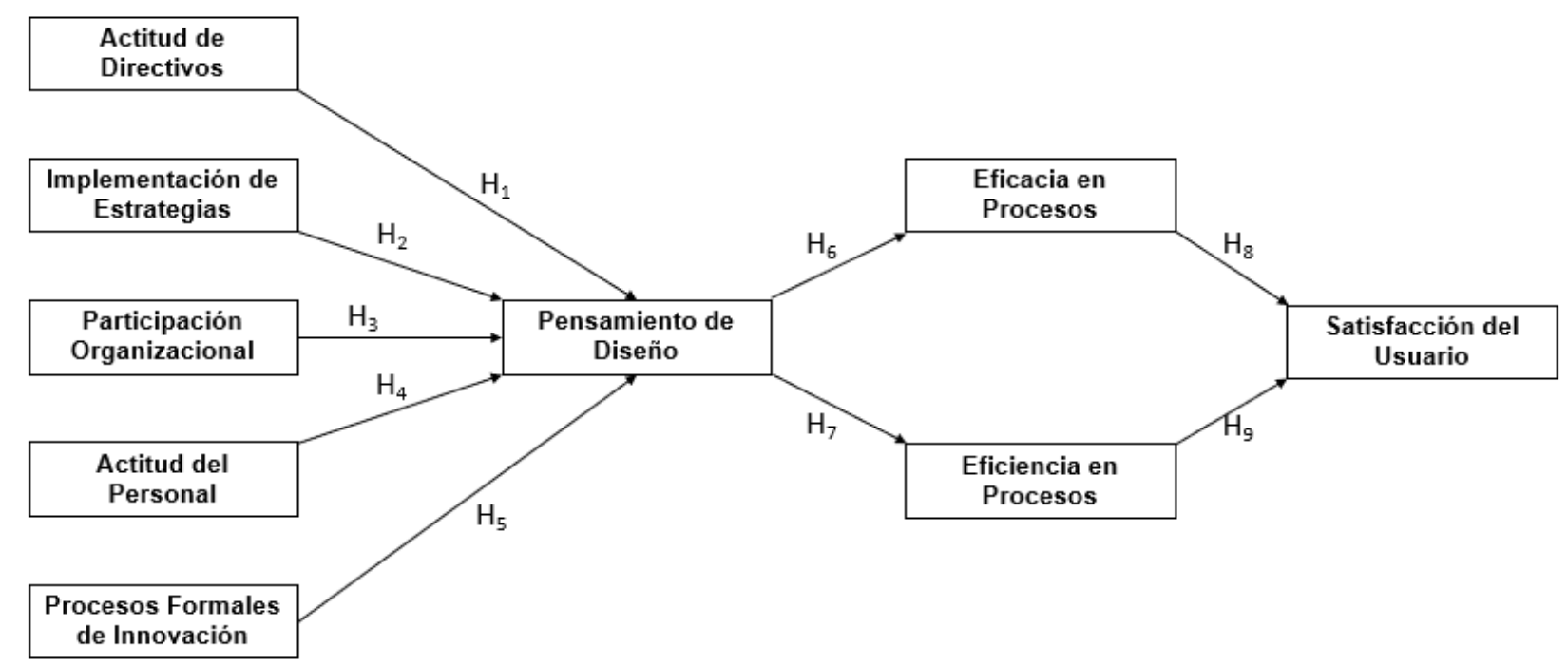

Fig. 1: Modelo causal satisfacción del usuario centrado en pensamiento de diseño

\section{METOdOLOGÍA}

El objetivo de esta investigación es desarrollar un modelo multivariado que permita analizar la influencia del pensamiento de diseño en la satisfacción de los usuarios de centros de salud familiar municipal, de la comuna de La Serena, Chile, además, de comprobar las hipótesis antes planteadas. Para el logro de estos objetivos, el método empleado para recoger la información es de carácter cuantitativo basado en una encuesta estructurada de 43 afirmaciones de tipo Likert, la que abarcó los siguientes ítems: actitud de los directivos, implementación de estrategias, participación organizacional, actitud del personal, procesos formales de innovación, orientación al pensamiento de diseño, eficiencia, eficacia y satisfacción del usuario.

El desarrollo del instrumento de evaluación fue realizado en conjunto a un panel de experto de la universidad de La Serena y a un equipo de expertos en gestión de salud pública pertenecientes a los centros de salud familiar de la ciudad de La Serena. El trabajo de campo se realizó de manera personal, entre mayo y noviembre 2018, se eliminaron datos que presentaban errores de medida e incongruencias, representados con una desviación típica de 0 . Los casos válidos ascendieron a 346 lo que representa el 36,42\% de los CESFAM de la comuna, se destaca también que la mayoría de los trabajadores son del género femenino (73,78\%), presentando un ingreso promedio mensual entre los US\$1000 y US\$1300. La Ficha Técnica de la investigación se presenta en Tabla 1.

Tabla 1: Ficha Técnica de Investigación

\begin{tabular}{|l|l|}
\hline Tipo de muestreo & Muestreo probabilístico \\
\hline Nivel de confianza & $95 \% ; \mathrm{z}=1.96 ; \mathrm{p}=\mathrm{q}=0.5$ (5\% error y $95 \%$ de confiabilidad) \\
\hline Tamaño muestral & 346 trabajadores \\
\hline Unidad de análisis & Centros de salud Cardenal Caro y Pedro Aguirre Cerda \\
\hline Método de recogida de la información & Cuestionario presencial de 43 afirmaciones \\
\hline Tipo de pregunta encuesta & Politómicas tipo Likert (1 a 5) y de selección \\
\hline Fecha realización del trabajo de campo & mayo y noviembre 2018 \\
\hline
\end{tabular}




\section{Fiabilidad Individual de los Indicadores}

Con la finalidad de evaluar la viabilidad del análisis factorial, se cuantificó el índice Kaiser-Meyer-Olkin (KMO) el que fue superior a 0,5 y la prueba de esfericidad de Bartlett (PEB) la que debe ser significativa $(p<0,05)$, (Williams et al., 2010), por lo que es factible realizar un análisis factorial. Para esto se utilizó el software SmartPLS en su versión 3.2.6. La fiabilidad de los indicadores se evaluó mediante el cálculo de los valores cuadráticos de las cargas externas, es decir, las cargas de los indicadores sobre su variable latente correspondiente, siendo un valor mínimo aceptable mayor a 0,4 (Damert et al., 2017). La tabla 2 evidencia que todos los indicadores son parte de los constructos.

Tabla 2: Fiabilidad Individual de los Indicadores

\begin{tabular}{|c|c|c|c|c|c|}
\hline Constructo & Indicador & Carga Factorial & Constructo & Indicador & $\begin{array}{c}\text { Carga } \\
\text { Factorial }\end{array}$ \\
\hline \multirow{6}{*}{$\begin{array}{l}\text { Actitud de los } \\
\text { Directivos } \\
\text { (ACTD) }\end{array}$} & ACTD01 & 0,834 & \multirow{5}{*}{$\begin{array}{l}\text { Pensamiento } \\
\text { de diseño } \\
\text { (DSTH) }\end{array}$} & DSTH01 & 0,832 \\
\hline & ACTD02 & 0,912 & & DSTH02 & 0,878 \\
\hline & ACTD03 & 0,895 & & DSTH03 & 0,808 \\
\hline & ACTD04 & 0,822 & & DSTH04 & 0,849 \\
\hline & ACTD05 & 0,787 & & DSTH05 & 0,848 \\
\hline & ACTD06 & 0,851 & \multirow{4}{*}{$\begin{array}{l}\text { Eficacia en } \\
\text { Procesos } \\
\text { (EFCA) }\end{array}$} & EFCA01 & 0,906 \\
\hline \multirow{3}{*}{$\begin{array}{c}\text { Implementación } \\
\text { de Estrategias } \\
\text { (IMPE) }\end{array}$} & IMPE01 & 0,857 & & EFCA02 & 0,949 \\
\hline & IMPE02 & 0,912 & & EFCA03 & 0,958 \\
\hline & IMPE03 & 0,877 & & EFCA04 & 0,880 \\
\hline \multirow{4}{*}{$\begin{array}{l}\text { Participación } \\
\text { Organizacional } \\
\text { (PARO) }\end{array}$} & PAR001 & 0,876 & \multirow{5}{*}{$\begin{array}{c}\text { Eficiencia en } \\
\text { Procesos } \\
\text { (EFCl) }\end{array}$} & EFCl01 & 0,838 \\
\hline & PARO02 & 0,873 & & EFCl02 & 0,715 \\
\hline & PARO03 & 0,854 & & EFCl03 & 0,825 \\
\hline & PARO04 & 0,879 & & EFCl04 & 0,880 \\
\hline \multirow{5}{*}{$\begin{array}{l}\text { Satisfacción del } \\
\text { Usuario (STSU) }\end{array}$} & STSU01 & 0,847 & & EFCI05 & 0,919 \\
\hline & STSU02 & 0,906 & \multirow{9}{*}{$\begin{array}{l}\text { Actitud del } \\
\text { Personal } \\
\text { (ACTP) }\end{array}$} & ACTP01 & 0,820 \\
\hline & STSU03 & 0,907 & & ACTP02 & 0,867 \\
\hline & STSU04 & 0,888 & & ACTP03 & 0,868 \\
\hline & STSU05 & 0,617 & & \multirow{2}{*}{ ACTP04 } & \multirow{2}{*}{0,796} \\
\hline \multirow{5}{*}{$\begin{array}{l}\text { Procesos } \\
\text { Formales de } \\
\text { Innovación } \\
\text { (PRFI) }\end{array}$} & PRFI01 & 0,768 & & & \\
\hline & PRFI02 & 0,818 & & \multirow{2}{*}{ ACTP05 } & \multirow{2}{*}{0,800} \\
\hline & PRFI03 & 0,882 & & & \\
\hline & PRFI04 & 0,772 & & \multirow{2}{*}{ ACTP06 } & \multirow{2}{*}{0,823} \\
\hline & PRFI05 & 0,786 & & & \\
\hline
\end{tabular}

\section{Fiabilidad del constructo}

Para evaluar la consistencia interna de los indicadores que miden los constructos reflectivos, se ha analizado la fiabilidad del constructo por medio del alfa de Cronbach el que debe ser estrictamente mayor a 0,7 (Bagozzi y Yi, 2012). El alfa de Cronbach para cada factor por separado no tiene en cuenta la influencia sobre la fiabilidad del resto de constructos. Por esta razón, se realiza el cálculo de la fiabilidad compuesta (IFC) la cual sí tiene en cuenta las interrelaciones de los constructos, además tiene como valor mínimo 0,7 (Bagozzi y Yi, 2012). La tabla 3 muestra que los constructos cumplen con los valores mínimos establecidos por la literatura.

Tabla 3: Fiabilidad y validez convergente de los constructos

\begin{tabular}{|l|c|c|c|}
\hline Constructo & $\begin{array}{c}\text { Alfa de } \\
\text { Cronbach }\end{array}$ & $\begin{array}{c}\text { Fiabilidad } \\
\text { Compuesta (IFC) }\end{array}$ & $\begin{array}{c}\text { Validez Convergente } \\
\text { (AVE) }\end{array}$ \\
\hline Actitud de los Directivos (ACTD) & 0,924 & 0,940 & 0,725 \\
\hline Implementación de Estrategias (IMPE) & 0,859 & 0,913 & 0,779 \\
\hline Participación Organizacional (PARO) & 0,894 & 0,926 & 0,758 \\
\hline Actitud del Personal (ACTP) & 0,910 & 0,930 & 0,688 \\
\hline Procesos Formales de Innovación (PRFI) & 0,865 & 0,903 & 0,650 \\
\hline Pensamiento de Diseño (DSTH) & 0,898 & 0,925 & 0,711 \\
\hline Eficacia en Procesos (EFCA) & 0,942 & 0,959 & 0,853 \\
\hline Eficiencia en Procesos (EFCI) & 0,893 & 0,921 & 0,702 \\
\hline Satisfacción del Usuario (STSU) & 0,891 & 0,922 & 0,706 \\
\hline
\end{tabular}




\section{Validez convergente y discriminante}

Para determinar la validez del constructo se realizan dos análisis: la validez convergente y la validez discriminante. La validez convergente expuesta en la tabla 3 , se comprueba a través de la varianza extraída media de los constructos, la que debe presentar un valor mínimo recomendado de 0,5 , lo que afirma que las variables explican más del $50 \%$ de la varianza total de sus constructos (Bagozzi y Yi, 2012). La validez discriminante presentada en la tabla 4, indica si un constructo es diferente a los otros, utilizando el criterio de que la raíz cuadrada de la varianza extraída media, la que para cada constructo debe ser mayor que sus correlaciones con todos los otros, es decir, el valor indicado en la diagonal debe ser estrictamente mayor al de filas y columnas (Bagozzi y Yi, 2012).

Tabla 4: Validez discriminante de los constructos

\begin{tabular}{|c|c|c|c|c|c|c|c|c|c|}
\cline { 2 - 12 } \multicolumn{1}{c|}{} & ACTD & IMPE & PARO & ACTP & FORP & DSTH & EFCA & EFCI & STSU \\
\hline ACTD & 0,851 & & & & & & & & \\
\hline IMPE & 0,603 & 0,883 & & & & & & & \\
\hline PARO & 0,522 & 0,686 & 0,871 & & & & & & \\
\hline ACTP & 0,416 & 0,520 & 0,530 & 0,830 & & & & & \\
\hline FORP & 0,595 & 0,692 & 0,715 & 0,572 & 0,806 & & & & \\
\hline DSTH & 0,593 & 0,569 & 0,664 & 0,529 & 0,706 & 0,843 & & & \\
\hline EFCA & 0,547 & 0,484 & 0,516 & 0,609 & 0,574 & 0,726 & 0,924 & & \\
\hline EFCI & 0,567 & 0,643 & 0,635 & 0,552 & 0,604 & 0,669 & 0,758 & 0,838 & \\
\hline STSU & 0,489 & 0,457 & 0,382 & 0,553 & 0,471 & 0,623 & 0,642 & 0,609 & 0,840 \\
\hline
\end{tabular}

\section{RESULTADOS}

Se planteó un análisis de ecuaciones estructurales para el contraste del modelo de relaciones propuesto (Fig. 1), que se desarrolló mediante el software SmartPLS, del cual se obtuvo también la varianza explicada y la validez predictiva del modelo.

\section{Coeficiente de determinación y validez predictiva del modelo}

El coeficiente de determinación de las variables endógenas o dependientes $\left(R^{2}\right)$ debe ser igual o mayor que 0,1 como valor mínimo (Falk y Miller, 1992). Por otra parte, el Test de Stone-Geisser $\left(Q^{2}\right)$, también denominado validación cruzada permite conocer la relevancia predictiva de un modelo estructural, la que debe adecuarse a predecir cada variable endógena latente (Hair et al., 2011). En el caso de que la $\mathrm{Q}^{2}>0$, indica que el modelo tiene relevancia predictiva (Chin, 1998). Como se aprecia en la tabla 5, todos los constructos presentan relevancia predictiva, además la satisfacción del usuario es explicada en un $69,7 \%$ gracias a la eficacia y la eficiencia de los procesos.

Tabla 5: Varianza explicada y validez predictiva del modelo

\begin{tabular}{|l|c|c|}
\hline Constructo & $R^{2}$ & $Q^{2}$ \\
\hline Pensamiento de Diseño & 0,641 & 0,513 \\
\hline Eficacia en Procesos & 0,527 & 0,443 \\
\hline Eficiencia en Procesos & 0,448 & 0,302 \\
\hline Satisfacción del Usuario & 0,697 & 0,556 \\
\hline
\end{tabular}

Bondad de ajuste del modelo

Esposito et al. (2010) sugieren un criterio global de bondad de ajuste para los modelos estructurales PLS, proponen que el índice de bondad de ajuste global se dé por medio de la raíz cuadrada de la multiplicación de la media aritmética del análisis de la varianza extraída (AVE) y la media aritmética del coeficiente de determinación $\left(R^{2}\right)$ de las variables endógenas o dependientes. Como se observa en tabla 6 , el índice de bondad de ajuste (GoF) del modelo de análisis es de 0,649 demostrando que se tiene un buen ajuste en el modelo de medida y en el modelo estructural, cumpliendo así con el criterio empírico de que la medida de bondad de ajuste debe variar entre 0 y 1, a mayor valor, mejor será el índice (Tenenhaus et al., 2005). 
Tabla 6: Modelo de ecuaciones estructurales: Análisis de relaciones causales y contraste de hipótesis.

\begin{tabular}{|c|c|c|c|c|c|}
\hline Hipótesis & Relaciones & Coeficiente PATH & Estadístico T & P-Value & Contraste \\
\hline H1 & ACTD $\rightarrow$ DSTH & 0,215 & 2,639 & 0,008 & Acepta \\
\hline H2 & IMPE $\rightarrow$ DSTH & 0,007 & 0,085 & 0,933 & Rechaza \\
\hline H3 & PARO $\rightarrow$ DSTH & 0,272 & 3,273 & 0,000 & Acepta \\
\hline H4 & ACTP $\rightarrow$ DSTH & 0,205 & 1,671 & 0,095 & Rechaza \\
\hline H5 & FORP $\rightarrow$ DSTH & 0,328 & 3,684 & 0,000 & Acepta \\
\hline H6 & DSTH $\rightarrow$ EFCA & 0,726 & 16,938 & 0,000 & Acepta \\
\hline H7 & DSTH $\rightarrow$ EFCl & 0,669 & 14,799 & 0,000 & Acepta \\
\hline H8 & EFCA $\rightarrow$ STSU & 0,442 & 4,308 & 0,000 & Acepta \\
\hline H9 & EFCI $\rightarrow$ STSU & 0,349 & 3,905 & 0,000 & Acepta \\
\hline
\end{tabular}

Relaciones causales y contraste de hipótesis

La metodología PLS no presume que la información está normalmente distribuida, lo que significa que, para evaluar la calidad del modelo completo, es necesario aplicar una técnica de re-muestreo no paramétrico llamado bootstrapping, la que involucra re-muestreo aleatorio con reposición de la muestra original, creando nuevas pseudo-muestras a partir de la muestra original con el objetivo de obtener los errores muestrales para testeo de hipótesis. La nueva muestra obtenida por este proceso permite la estimación de coeficientes en la metodología PLS-SEM para testear su significancia estadística (Hair et al., 2011). Esta técnica, ofrece el cálculo del error estándar de los parámetros, donde se debe verificar la condición del estadístico T>=1,96 para poder determinar su nivel de significancia (Kwong, 2013). La tabla 6 indica el contraste de hipótesis.

\section{DISCUSIÓN}

La primera contribución de esta investigación, es la confirmación del carácter multidimensional del pensamiento de diseño como variable a gestionar en los CESFAM de la comuna de La Serena, el cual logra ser explicado en un $64,1 \%$ por variables causales como; actitud de los directivos, participación organizacional, y formalización de procesos innovadores, siendo esta última la más significativa acorde a los valores de los coeficientes estandarizados, en este ámbito Schultz et al. (2013) han demostrado empíricamente que la formalización de los procesos conduce al éxito. Desde este punto de vista se ratifica lo expresado por Roberts et al., (2016) quienes establecen que formalizar la innovación en el diseño de los procesos ayuda a establecer directrices para la innovación y el intercambio, mejorando así la capacidad de la organización para aplicar los conocimientos y mejorar la cooperación entre los empleados.

El pensamiento de diseño en los CESFAM debe estar centrado en la innovación tanto de los procesos, como en las prácticas del personal y de los directivos, quienes deben adoptar nuevas prácticas en torno a un nuevo procedimiento y proceso, donde el pensamiento de diseño, como metodología y sus paradigmas inherentes, podría acelerar las innovaciones cuando la etapa de ideación se estanca por falta de ideas o conflictos entre los principales actores claves, innovaciones imprescindibles en la gestión de la salud primaria chilena, considerando lo expresado por la (OCDE, 2016). Esto se debe en parte al bajo gasto que destina Chile en materias de salud, sólo USD 1.977 per cápita, muy por debajo de los USD 4.003 per cápita que gastan en promedio los países pertenecientes a las OCDE (OCDE, 2017), lo que ratifica que el compromiso estratégico del estado chileno también se evidencia con un mayor presupuesto que permita reducir la brecha anterior, incorporando nuevas tecnologías, e innovaciones que mejoren los tradicionales procesos y procedimientos desarrollados por los organismos de salud en Chile.

La investigación dejó en evidencia que la satisfacción del usuario es explicada en un $69,7 \%$ por la eficiencia y eficacia de los procesos del CESFAM, cuyos diseños se basan en el pensamiento de diseño, potenciando así una atención centrada en las personas, y no sólo en los pacientes, lo que confirma Medeiros et al. (2010), al referirse que el usuario, al buscar un centro de salud, espera que su problema sea resuelto individual $o$ colectivamente, que el conjunto de acciones de salud sea beneficioso y que las acciones sean efectivas y satisfactorias. Si bien lo anterior representa un alto valor de explicación de la satisfacción de los usuarios de Ios CESFAM, es igualmente recomendable incorporar nuevas variables para lograr una mayor explicación del modelo causal propuesto, estas pueden ser: Imagen institucional, disponibilidad de tecnología en la atención, accesibilidad, credibilidad de los conocimientos técnicos que tiene el personal, formación del personal clínico, fallas estructurales del sistema, asimetría emocional entre el profesional y el paciente, la consistencia entre los valores del personal y de la organización de salud, entre otros. 
Los resultados univariantes de esta investigación evidencian que en los CESFAM no se gestionan sistémicamente las seis variables que influyen en la satisfacción del usuario de los centros de salud familiar, pues sus niveles se encuentran en un rango bajo a medio, en el caso de eficacia de los procesos, los resultados alcanzan un nivel de un 53,5\%; eficiencia de los procesos $57,4 \%$; formalización de los procesos de innovación un $63,7 \%$; actitud de los directivos $59,1 \%$ y participación del personal $66,4 \%$, generando en su conjunto bajos niveles de satisfacción por parte del usuario equivalente a un $58,5 \%$. Estos resultados empíricos son similares a los obtenidos por Valenzuela y Pezoa (2015), quienes, en su estudio sobre salud pública chilena, obtuvieron $64,3 \%$ de satisfacción por parte del usuario hacia los sistemas de salud. La investigación ratifica que la satisfacción del usuario es un criterio de diseño vital para los sistemas sostenibles, demostrado además que esta variable es un indicador fiable para medir la calidad del servicio referente a lo clínico (Woodward et al., 2017).

Los centros de salud familiar latinoamericanos deben contar con profesionales de valores éticos consolidados cuyos procesos de innovación tengan como pilar la ética. En Canadá por ejemplo se ha publicado la guía Ethics in Family Medicine elaborada por el Comité Ético del Colegio de Médicos de Familia de Canadá, destinada a los médicos de familia. La publicación de esta guía surge por la falta de disponibilidad de expertos en ética, especialmente en unidades docentes rurales (Pérez-García, 2015).

Roberts et al. (2016), sostienen que el pensamiento de diseño en Estados Unidos debe incorporarse como una competencia central dentro de la administración de la salud, ofreciendo un complemento valioso a los conjuntos de habilidades disciplinarias fundamentales, como la planificación estratégica, la gestión de operaciones, la gestión del personal y la mejora de procesos, como una forma de ayudar a navegar e impulsar soluciones innovadoras a los problemas de la atención médica. En su investigación concluyen que la demora en el ritmo del cambio y la innovación dentro del sistema de salud actual no se deben a la falta de visión, esfuerzo o incluso recursos; sino más bien surge de los intentos de rehacer un modelo de atención médica que nunca fue diseñado para hacer las cosas que ahora se le piden. Estos resultados se encuentran en línea a lo obtenido en esta investigación, pues se debe reestructurar el modelo de atención entregada a los usuarios de los servicios de Salud Familiar por medio de la metodología del pensamiento de diseño cuyo foco debe ser la satisfacción final del usuario.

Los gobiernos locales deben instalar el pensamiento de diseño, como metodología y paradigma, en cada de uno de los centros de atención primaria de salud, lo que generará una mayor satisfacción a los usuarios debido a una mejor calidad en el servicio, ratificando lo expresado por Lacerda et al. (2017), a mayor calidad de servicio, mayor será la satisfacción del usuario. En esta misma línea el estado y los centros de salud de familiar deben contar con un personal

\section{CONCLUSIONES}

A partir de los resultados empíricos se obtiene las siguientes conclusiones:

1. Los directivos de los centros de salud primaria deben enfocarse en instalar la metodología y paradigma del pensamiento de diseño, centrándose en la formalización de procesos innovadores, permitiendo la sistematización y ejecución pertinente de los mismos en los CESFAM. Por ello, los líderes deben aprender a desarrollar en sus colaboradores las capacidades para influir continuamente en la cultura organizacional, aumentando la sintonía de actitudes y valores entre los integrantes del CESFAM y los valores corporativos que la organización busca instalar.

2. La participación organizacional es indispensable para que la metodología del pensamiento de diseño impulse un diseño de procesos eficientes y eficaces, gracias a la innovación centrada en sus usuarios la que se genera y aplica por el personal, independiente de su profesión, lo que hace imprescindible capturar la "voz y vivencias" de sus clientes externos (pacientes, familiares y entorno social) de un CESFAM. Para esto se debe contar con un personal con competencias multidisciplinarias, pues instalar pensamiento de diseño para mejorar el sistema de salud, requiere de dotarlos de un enfoque más humanista y no sólo técnicofuncional, así en parte se podrán superar los retos que tiene por delante la gestión de la salud pública, para adaptarse y/o evolucionar a un paradigma de servicio centrado realmente en las personas.

3. La gestión sistémica de los centros de salud familiar también se debe enfocar en fortalecer la eficiencia y eficacia de su personal, fortaleciendo su participación a través de procesos innovadores. Así se aporta a la satisfacción del usuario (cliente externo). Este desafío es urgente si se considera al menos el creciente empoderamiento de los pacientes, que serán cada vez menos percibidos como usuarios, y además, las tres fuentes generadoras de tensión y/o conflictos funcionales y disfuncionales. A saber, la falta de empatía entre; el personal clínico con los pacientes, los profesionales clínicos con los profesionales de gestión y la atención primaria con la atención especializada. 


\section{AGRADECIMIENTOS}

Los autores agradecen el apoyo entregado por la Universidad de La Serena para terminar exitosamente esta investigación.

\section{REFERENCIAS}

Bagozzi, R y Y. Yi, Specification, evaluation, and interpretation of structural equation models, Journal of the Academy of Marketing Science, 40(1), 8-34 (2012).

Bhui, K., Quality improvement and psychiatric research: Can design thinking bridge the gap? The British Journal of Psychiatry, 210(5), 377-378 (2017).

Brown, T., Design Thinking. Harvard Business Review, 86(6), 1-10 (2008).

Carlgren, L., Design Thinking as an enabler of innovation: Exploring the concept and its relation to building innovation capabilities, doctoral degree thesis, Chalmers University of Technology, Gotemburgo, Suecia (2013).

Castillo, M., A. Álvarez y S. Cabana; Design Thinking: Como guiar a estudiantes, emprendedores y empresarios en su aplicación. Ingeniería Industrial, 35(3), 301-311 (2014).

Chin, W., The Partial Least Squares Approach to Structural Equation Modeling, in G.A. Marcoulides [ed.], 295-336, Modern Methods for Business Research, Mahwah, NJ: Lawrence Erlbaum Associates, (1998).

Clínicas de Chile A.G.; Innovación en Gestión de Salud, 22, 1-5 (2009).

Da Costa, S., D. Páez y otros 3 autores, Factores favorables a la innovación en las Organizaciones: Una integración de Meta-análisis, Journal of work and organizational Psichology, 30(2), 67-74 (2014).

Damert, M., A. Paul y R. Baumgartner, Exploring the determinants and long-term performance outcomes of corporate carbon strategies, Journal of Cleaner Production, 160(1), 123-138 (2017).

Dülfer, N., Designing for Success: Investigating Design as a driver for innovation, doctoral degree thesis. Maastricht University, Mastricht, Países Bajos (2014).

Esposito, V., W. Chin, J. Henseler y H. Wang, Handbook of Partial Least Squares: Concepts, Methods and Applications, New York, England (2010).

Falk, R. y N. Miller, A primer for soft modeling, Ohio, United States: University of Akron Press (1992).

Hair, J., C. Ringle, y M. Sarstedt, PLS-SEM: Indeed a Silver Bullet, Journal of Marketing Theory and Practice, 19(29), 139151 (2011).

Kessler, I., P. Heron y K. Spilsbury, Human resource management innovation in health care: the institutionalisation of new support roles, Human Resource Management Journal, 27(2), 228-245 (2017).

Kwong, K., Partial Least Squares Structural Equation Modeling (PLS-SEM) Techniques Using SmartPLS, Marketing Bulletin, 24(1), 1-32 (2013).

Lam, R. y P. Hernández, Los términos: eficiencia, eficacia y efectividad ¿son sinónimos en el área de la salud? Revista Cubana de Hematología, Inmunología y Hemoterapia, 24(2), 1-6 (2008).

Lacerda, A., L. Bezerra, L. dos Santos y A. Gondim, Factors associated with user satisfaction regarding treatment offered in Brazilian primary health care, Cadernos de Saúde Pública, 33(2), 1-15 (2017).

Medeiros, F., G. Araújo-Souza, A. Albuquerque-Barbosa y I. Clara-Costa, Basic health unit embracement: focusing on user satisfaction, Revista Salud Pública, 12(3), 402-413 (2010).

McKee, D., C. Simmers y J. Licata, Customer Self-Efficacy and Response to Service, Journal of Service Research, 8(3), 207-220 (2006).

Méndez, M. y S. Rondón, Introducción al Análisis Factorial Exploratorio, Revista Colombiana de Psiquiatría, 41(1), 197207 (2012).

Miranda, I. y A. Astorga, Relación entre gestión del conocimiento e innovación, Sciences for Innovation, 3(4), 34-41 (2013).

Nebot, M., C. Ariza, J. Villalbí y A. García-Altés, Evaluación de la efectividad en salud pública: fundamentos conceptuales y metodológicos, Gaceta Sanitaria, 25(1), 3-8 (2011).

Nikulin, C., C. Arata, R. Stegmaier y F. Soto, Identificación de Factores Críticos de Innovación para Modelo de Medición de Innovación por Etapas que sean representativos para los Indicadores Internacionales de I + D + I mediante la Formación de Clúster, Sciences for Innovation, 1(1), 19-34 (2011).

OCDE, Panorama de la Sociedad 2016, Un primer plano sobre los jóvenes (2016).

OCDE, Health at a Glance 2017 - OECD Indicators. (2017)

Oliver, R., Satisfaction: A Behavioral Perspective on the Consumer, New York, USA: McGraw-Hill (1997).

Pérez-García, R., La competencia en bioética: eje fundamental en la formación de residentes de Medicina Familiar y Comunitaria, Educación Médica, 19(4), 235-240 (2015). 
Pezoa, M., Propuesta Metodológica para Medir Satisfacción en Usuarios de Consultorios Públicos. (2011)

Roberts, D., F. Piller y D. Luettgens, Mapping the impact of social media for innovation: The role of social media in explaining innovation performance in the PDMA comparative performance assessment study, Journal of Product Innovation Management, 33(S1), 117-135 (2016).

Roberts J., Fisher T., Trowbridge M. y Bent C, A design thinking framework for healthcare management and innovation, Academic Radiology, 4(1), 11-4 (2016).

Schultz, C., S. Salomo, U. de Bretani y E. Kleinschmidt, How formal control influences decision making clarity and innovation performance, Journal of Product Innovation Management, 30(3), 430-447 (2013).

Shanker, R., R. Bhanugopan, B. Van der Heijden y M. Farrell, Organizational climate for innovation and organizational performance: The mediating effect of innovative work behaviour, Journal of Vocational Behavior, 100(1), 67-77 (2017).

Tenenhaus, M., V. Esposito, Y. Chatelin y C. Lauro, PLS path modeling, Computational Statistics \& Data Analysis, 48(1), 159-205 (2005).

Valenzuela, P. y M. Pezoa, Estudio de opinión a usuarios del sistema de salud, reforma y posicionamiento de la superintendencia de salud, (2015).

Williams, B., T. Brown y A. Onsman, Exploratory factor analysis: A five-step guide for novices, Journal of Emergency Primary Health Care, 8 (3), 1-13 (2010)

Wintrup, J., The changing landscape of care: Does ethics education have a new role to play in health practice?, BMC Medical Ethics, 16(1), 22 (2015).

Woodward, F., G. Berry y Y. Bucci, A systematic review of factors associated with service user satisfaction with psychiatric inpatient services, Journal of Psychiatric Research, 92(1), 81-93 (2017). 
